\title{
Lack of Relation of Survivin Gene Expression with Survival and Surgical Prognostic Factors in Endometrial Carcinoma Patients
}

\author{
Rifat Taner Aksoy ${ }^{1 *}$, Ahmet Taner Turan ${ }^{1}$, Nurettin Boran ${ }^{1}$, Aytekin Tokmak ${ }^{3}$, \\ Betul Zuhal Isikdogan ${ }^{1}$, Mehmet Dogan ${ }^{2}$,Hakki Gokhan Tulunay ${ }^{1}$
}

\begin{abstract}
Background: The relation of survivin gene expression to survival and surgical prognostic factors in the patients with endometrial carcinoma is unclear. Materials and Methods: In this study, 62 cases who were operated due to endometrial carcinoma were investigated between 2003 and 2011 in the the gynecological oncology clinic of Female Disease Training and Investigation Hospital of Etlik Zubeyde, Hanim, Turkey. Clinical and surgical prognostic factors were investigated by screening the records of these cases. With the standard streptavidin-biotin immune peroxidase method, cytoplasmic and nuclear expression of survivin was investigated in sections with specific antibodies (1:100, diagnostic Bio Systems, USA) primer. The aim was to elucidate any relation between survivin expression and defined prognostic factors and survival. Results: There was no statistically significant relationship between cytoplasmic and nuclear indexes identified for survivin and age, body mass index, the levels of preoperative hemoglobin, platelet and $\mathrm{Ca}$ 125, stage, grade, lymph node meastasis, the number of metastatical lymph nodes (total, paraaortic and pelvic), myometrial invasion, serosal invasion, adnexal involvement, the presence of acid in the first diagnosis, the involvement of omentum, the adjuvant treatment application of the cases, the presence of recurrence and rate of mortality $(p>0.05)$. Statistical significance was noted for the presence of advanced stage lymph node metastasis (pelvic, paraaortic, pelvic and paraaortic), serosal involvement, positive cytology, lymph vascular space invasion, intra abdominal metastasis, and omentum involvement. When investigated the relation between cytoplasmic and nuclear survivin indexes and total survival, the result was not statistically significant $(\mathbf{p}>\mathbf{0 . 0 5})$. Conclusions: In our study, there was no statistically significant relationship between the rates of cytoplasmic and nuclear survivin expression with identified prognostic factors and total or non-disease survival.
\end{abstract}

Keywords: Survivin - endometrial carcinoma - survival - Turkey

Asian Pac J Cancer Prev, 15 (16), 6905-6910

\section{Introduction}

Endometrial carcinoma is a neoplasm characterized by the apparent hyperplasia and anaplasia of glandular elements with the invasion of endometrial tissue in to stroma, myometrium and vascular spaces. In developed countries, endometrial cancer is the commonest malign tumor of the female genital system. And the cases are the females with the advanced age who are generally examined symptomatically stage 1 (Silverberg et al., 1990) The dualistic modal based on clinical pathological correlations in endometrial carcinogenicity has been identified. According to this model, Type 1 carcinomas are related to the estrogen-dependent pathway. EC is the fourth common cancer among women and had a prevalance of 6 to $9 \%$ (Rowlands et al., 2011). Histologically, there is hyperplastic endometrial in the backround of most type 1 neoplasms. (Silverberg et al., 1990; Felix et al., 2010) And they are the low grade tumors. Type 1 endometrial adenocarcinomas reveal MSI and PTEN, PIK3CA, K-KAS, CTNNB1 ( $\beta$-catenin) mutations, Type 2 endometrial adenocarcinomas reveal p53 mutations and chromosomal instability, the loss of heterozygosity and Her2/neuamplication. Independently from estrogen, Type 2 carcinomas develop in the atrophical endometrial background. They are high graded neoplasm and are in serous or lucid cell morphology.

After the advent of immune-histochemical studies, the new terms have been proposed in identifying the prognosis of EC and the new prognostic factors have been defined. Baser et al (2013) reported that spermassociated antigen 9 may be a promsing marker in early diagnosis of EC. Nowadays, the effects of oncogenes, ploidi and molecular indicators on the prognosis are still 
researched. Survivin is a bifunctional protein suppressing apopitosis which controls the cell division and is also referred to as programmed cell death. Survivin, a member of apoptosisinhibitor gene family, is expressed in G2/M phase of the cell cycle. Though it is not almost identified in most normal tissues with terminal differentiation, the newly researches have demonstrated that survivin is extremely expressed in various cancer types (Altieri et al., 1999; Karimi et al., 2013). In several studies, the extreme expression of survivin is related to bad prognosis in urinary bladder cancer (Srivastava et al., 2013), cervix uteri cancer (Cao et al., 2014), colorectal cancer (Yuan et al., 2012) and breast cancer (Damitri et al., 2014).

In a study, Okada et al (2001) reported both cytoplasmic and nuclear survivin expression in the stomach cancers and determined that cytoplasmic survivin expression was related to the bad prognosis. In multi-variable analysis, it has been pointed out that nuclear survivin expression would be able to be a good prognostic factor. The available data associated with the prognostic effect of survivin is paradoxical in endometrial carcinoma. In retrospective studies, the negative indicators of the disease progression, increased recurrence rate and reduced longevity associated with increased resistance to the treatment were revealed in the patients having survivin expression. In several studies survivin expression was found to be referred to bcl- 2 or low apoptotic index. Therefore, the detection of survivin protein in immuno-hystocemical or tumor samples by RTPCR may be the initial indicator of the aggressive disease. And it may confirm more detailed follow-up protocols or alternative treatment regimes.

In our study, our aim is to elucidate the relation between defined prognostic factors with survivin expression and survival in 62 endometrial carcinoma cases as a result of data obtained from the analysis.

\section{Materials and Methods}

This study includes 62 operated cases because of endometrial carcinoma between 2003-2011, in the Female Disease Training and Investigation Hospital of Etlik Zubeyde Hanim, the clinic of gynecological oncology. The records and pathology reports of these cases were searched. Hemotoxylin-Eosin stained preperates in the cases were reconsidered. For all cases, the block sunk into paraffin and fixed in formalin of $10 \%$ which belonged to the tissue sampling the tumor in the best manner was selected. By placing in the slides covered by poli-L-lysine, the cross-sections in 5 micron thickness prepared from the selected blocks were kept in the room temperature for immune-histochemical stain.

Cytoplasmic and nuclear survivin expressions in tumor tissue were assessed immune-histochemically. The spectrum of cytoplasmic stain was scored from 0 to 4 for survivin. $>75 \%$ positive (score 4 ), $50-75 \%$ positive (score 3 ), $25-50 \%$ positive (score 2 ), 1-25\% positive (score 1 ) and negative (score 0 ). Additionally, the density of the cytoplasmic stain was scored from 1 to 3 ( 1 poor, 2 moderate, 3 severe). And also, the spectrum and density of nuclear stain for survivin were scored similarly. If the final score obtained by the multiplication of the spectrum (s) and density (d) scores for all samples was assessed as negative, poor positive and strong positive respectively (Lurain et al., 1991). Normal stomach mucosa's biopsy was used as the positive control.

The statistical assessments of obtained data were performed by SPSS (Scientific Package for Social Sciences) 20.0 programme, Windows 7 operation system. In the assessments,Mann-Whitney U Test was used for the comparison of the variables identified by the measurement in the situations with two categories. For more than two categories, Kruskall-Wallis unidirectional variant analysis and for the quantitative variable relations, Spearman Rank Correlation Analysis were used. In addition, for time-dependent changes of survival and recurrence situations, Kaplan-Meier survival analysis and for the research of the factors influencing survival and recurrence, Cox's Regression Analysis were used. The statistically significant range was accepted as 0.05 .

\section{Results}

The ages of the cases during the diagnosis were $55.4 \pm 8$ and ranged between 31 and 75. Body Mass Index was $33.6 \pm 5.4$ and ranged between 19 and 50. While 44(70\%) patients were in the postmenopausal period, $28(30 \%)$ patients were in premenopausal period. As on additional disease, $37 \%$ of the cases had only hypertension. $7.4 \%$ had only diabetes mellitus and $19.2 \%$ had both hypertension and diabetes mellitus. The pathological investigation results of probe curettage materials performed primer presurgery were reported as endometrial adenocarcinoma in all cases. Maximal, optimal and suboptimal debulkings were provided in 59, 2 and 1 cases respectively. During the primary surgery in 55(88.7) cases, pelvic and paraaortic lymphadenectomy were performed. Omentectomy was also performed in the same cases during primary surgery. The number of total removal lymph node was $65.8 \pm 20.4$. the number of removal pelvic lymph node was $44.5 \pm 13.9$ and the number of removal paraaortic lymph node was $21.3 \pm 10$. While the hemoglobin platelet levels were measured in all patients preoperatively, 46 of the patients had CA125 values. In the cases mean hemoglobin levels, mean platelet values and median CA125 values were $11.9 \pm 2.1 \mathrm{mg} / \mathrm{dl}, 330145 \pm 109355 / \mathrm{mm}^{3}$ and $26.8 \mathrm{v}(3.1-473)$ $\mathrm{IU} / \mathrm{ml}$ respectively. The clinical and surgical pathological features of the patients are shown in details in Table 1.

In all cases, the postoperative pathology results were reported as endometrioid type adenocarcinoma of endometrial. The surgical stages of the cases were stage according to FIGO 2009 staging criteria. For this reason, $10(16.1 \%), 6(9.7 \%), 14(22.6 \%), 6(9.7 \%), 1(1.6 \%)$, $6(9.7 \%), 7(11.3 \%)$ and $12(19.4 \%)$ patients were classified as stage 1a, stage $1 \mathrm{~b}$, stage 2 , stage $3 \mathrm{a}$, stage $3 \mathrm{~b}$, stage $3 \mathrm{c} 1$, stage $3 \mathrm{c} 2$ and stage $4 \mathrm{~b}$ respectively. In all cases, histological grade ranges were grade 1 for $27(43.5 \%)$, grade 2 for 23(37.1\%) and grade 3 for 12(19.4\%). The average largest diameter of the tumor was measured as $45.6 \pm 18.8 \mathrm{~mm}$. The myometrial invasions of $19(30.6 \%)$ cases were less than $1 / 2$, whereas those of $43(69.4 \%)$ cases were equal or more. $6(9.7 \%)$ of the cases had serosal involvement. Lymph vascular space invasion 
was observed positively in $28(45.2 \%)$ cases. Total $17(27.4 \%)$ cases had adnexal involvement. While tubal involvement only detected positively in $2(3.2 \%)$ of the cases, $10(16.1 \%)$ cases had only over involvement and $5(8 \%)$ had both over and tubal involvement. At the time of the first diagnosis, the presence of the acid was detected in $3(4.8 \%)$ cases. In primary surgery, in the abdominal cytological sampling performed in all cases, malign cytology was observed in $9(14.5 \%)$ cases. $11(17.7 \%)$ cases had omentum involvement. During the primary surgery, tumor was observed within the other organs in the abdomen in 12(19.4\%) cases. Appendectomy was added to the surgery in $8(12.9 \%)$ cases and the pathology results in the half of these were reported as tumor-positive. The adjuvant treatment was performed in primer postsurgery for $45(72.6 \%)$ cases. Only radiotherapy for 26(57.8\%), only chemotherapy for $10(22.2 \%)$, radiotherapy after the chemotherapy for $3(4.8 \%)$, concomitant chemotherapy and radiotherapy for $3(4.8 \%)$ and chemotherapy subsequently radiotherapy and again chemotherapy, that is, sandwich treatment for $3(4.8 \%)$ cases were performed. The disease recurrence was seen in $13(21 \%)$ cases. Mean recurrence time was computed as $13.6 \pm 6.3$ months. The recurrence areas were occurred in the lung for $2(15.3 \%)$, in the liver for $2(15.3 \%)$, in vaginal cuff for $1(7.6 \%)$ and in the organs within abdomen for $8(61.5 \%)$ cases. Mean CA125 value during the recurrence was58.7 and ranged between 3.9 and $145 \mathrm{IU} / \mathrm{ml}$. After recurrence, the treatment was performed for all patients. While 11 patients received chemotherapy, 2 patients were primarily operated and subsequently received chemotherapy. While progression was observed in $11(84.6 \%)$ cases in spite of the treatment, the clinical partial response for $1(7.6 \%)$ case and clinical complete response for $1(7.6 \%)$ case were obtained.

The death was seen in $13(21 \%)$ cases. While 12(92.6) of these deaths occurred depending on the disease, it occurred in 1(7.6\%) case due to the cause apart from the disease. Mean death time was computed as $20.9 \pm 10.9$ months. The other 49(79\%) cases lived as non-diseased. Mean follow-up time of the cases was 40.9+30.1 months.

Cytoplasmic and nuclear survivin expression results detected by the immunechemical investigation in the cases were presented in details in Table 2. The statistical assessments of these results were presented in Table 3 .

When data obtained by the measurement of survivin expression were investigated, the presence of cytoplasmical stain was detected. It was seen that the stain spectrum in $45(72.5 \%)$ cases were above $50 \%$ commonly accepted in literature. When the spectrum of nuclear staining in the cases was investigated, it was observed that there was no stain in $16(25.8 \%)$ cases. In the majority of the cases, it was seen that nuclear staining was occurred in the focal level (43 cases; 69.4\%). While it was observed that the density of cytoplasmic survivin expression was mostly in poor or moderate intensity (56 cases; $90.3 \%$ ), it was seen that nuclear survivin expression density was mostly in moderate intensity (34 cases; 74\%). According to the indexes computed by taking into account of the staining spectrum and density of the cells, the median value for the index of cytoplasmic survivin expression was detected as 4(1-12) which is the threshold value used for poor and severe discrimination. Median value for the index of nuclear survivin expression was computed as 2(0-6). The relation between clinical and surgical pathological factors of the cases and cytoplasmic and nuclear expression rates of surviving was investigated in details by taking into account of the indexes.

Mean life time of the cases was computed as $84.04 \pm 6.36$. It was detected that the life possibilities(survival possibilities) were $96.6 \%$ for one year, $83.4 \%$ for two years, $72.7 \%$ for three years and $69.4 \%$ for four years. The relationships between total survival and data which have prognostic importance were seen in Table 4 and total survival graphic of the cases was presented in Figure 1. When the relationship between total survival

Table 1. The Clinical and Surgical Pathologic Features of the Patients

\begin{tabular}{|c|c|c|c|}
\hline Clinical and surgical path & logic features & $\mathrm{n} / \mathrm{means}$ & $\% /$ median \\
\hline Age & & 55.48 & $(31-75)$ \\
\hline Preoperative CA $125(\mathrm{IU} / \mathrm{n}$ & & 61 & 26,8 \\
\hline The type of tumor & Endometrioid & 62 & 100 \\
\hline Stage & Stage 1a & 10 & 16.1 \\
\hline & Stage $1 b$ & 6 & 9.7 \\
\hline & Stage 2 & 14 & 22.6 \\
\hline & Stage $3 a$ & 6 & 9.7 \\
\hline & Stage $3 b$ & 1 & 1.6 \\
\hline & Stage $3 c 1$ & 6 & 9.7 \\
\hline & Stage $3 c 2$ & 7 & 11.3 \\
\hline & Stage $4 b$ & 12 & 19.4 \\
\hline Grade & 1 & 27 & 43.5 \\
\hline & 2 & 23 & 37.1 \\
\hline & 3 & 12 & 19.4 \\
\hline Lymph node dissection & Performed & 55 & 88.7 \\
\hline & Not performed & 7 & 11.3 \\
\hline Omentectomy & Total & 30 & 56.2 \\
\hline & İnfracolic & 18 & 39.6 \\
\hline & Biopsy & 7 & $4-2$ \\
\hline Omentum metastasis & Yok & 44 & 20 \\
\hline & Var & 11 & 80 \\
\hline Acide & Yok & 59 & 95.2 \\
\hline & Var & 3 & 4.8 \\
\hline Peritoneal cytology & Negative & 53 & 85.5 \\
\hline & Positive & 9 & 14.5 \\
\hline Adnexal metastasis & Negative & 45 & 72.6 \\
\hline & Positive & 17 & 27.4 \\
\hline Myometrial invasion & $<1 / 2$ & 19 & 30.6 \\
\hline & $\geq 1 / 2$ and no-serosal inv & vasion & \\
\hline & & 37 & 59.6 \\
\hline & Serosal invasion & 6 & 9.7 \\
\hline Cervical invasion & Yok & 37 & 59.7 \\
\hline & Var & 25 & 40.3 \\
\hline Lymph vascular space & Negatif & 34 & 54.8 \\
\hline invasion & Pozitif & 28 & 45.2 \\
\hline Lymphadenectomy & Performed & 55 & 88.7 \\
\hline & Not performed & 7 & 11.3 \\
\hline The number of lymph & Total & 65.8 & 68 \\
\hline & & & $(17-105)$ \\
\hline nodes removed & Paraaortic & 21.3 & $20(2-45)$ \\
\hline & Pelvic & 44.4 & $44(8-76)$ \\
\hline The presence of lymph & Total & 22 & 35.5 \\
\hline node metastasis & Paraaortic & 15 & 27.3 \\
\hline & Pelvic & 18 & 29 \\
\hline The number of metastatic & Total & 6.8 & $3,5(1-34)$ \\
\hline lymph nodes & Paraaortic & 5.87 & $2(1-30)$ \\
\hline & Pelvic & 3.5 & $3(1-11)$ \\
\hline İntra abdominal & Negative & 50 & 80.6 \\
\hline metastasis b & Positive & 12 & 19.4 \\
\hline Primary surgical results & Suboptimal debulking & 1 & 1.6 \\
\hline & Optimal debulking & 2 & 3.2 \\
\hline & Maksimal debulking & 59 & 95.2 \\
\hline
\end{tabular}

Asian Pacific Journal of Cancer Prevention, Vol 15, 2014 


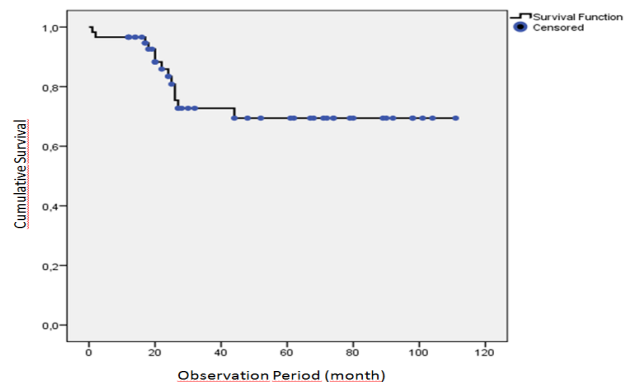

Figure 1. Total Survival Graphic of the Cases

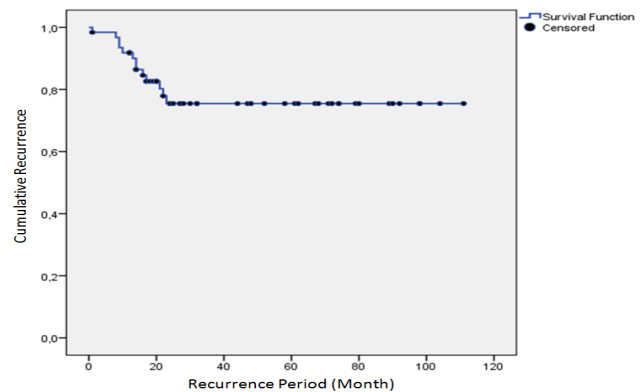

Figure 2. Non-disease Survival Graphic of the Cases

Table 2. The Results of Nuclear Survivin Expression

\begin{tabular}{lcrr}
\hline The assessment of nuclear survivin & Groups & $\mathrm{n}$ & $\%$ \\
\hline The spectrum of nuclear survivin expression & & \\
No staining & 16 & 25.8 \\
$\% 1-25$ & 43 & 69.4 \\
$\% 26-50$ & 3 & 4.8
\end{tabular}

The density of nuclear surviving expression

$\begin{array}{lrr}\text { Poor } & 6 & 13 \\ \text { Moderate } & 34 & 74 \\ \text { Severe } & 6 & 13\end{array}$

The index of nuclear survivin expression

\begin{tabular}{rrr}
0 & 16 & 25.8 \\
1 & 6 & 9.7 \\
2 & 33 & 53.2 \\
3 & 4 & 6.5 \\
4 & 1 & 1.6 \\
6 & 2 & 3.2 \\
\hline
\end{tabular}

and age, grade, preoperative hemoglobin, platelet and CA125 levels, adnexal involvement and the level of myometrial invasion was investigated, there was no statistically significances in the results $(p>0.05)$. When the relationship between total survival and advanced stage lymph node, the presence of metastasis (pelvic, paraaortic, pelvic and paraaortic), serosal involvement, cytology positivity, lymph vascular space invasion, the presence of intraabdominal metastasis, omentum involvement, the presence of acid in the first diagnosis and the presence of recurrences was investigated, the results obtained were statistically significant $(\mathrm{p}<0.05)$. While the indexes of cytoplasmic and nuclear survivin and total survival rates were investigated, advance stage cases (stage $3 \mathrm{a}$ and above) were considered a separate subgroup.

As a result of the statistical investigation carried out in this subgroup including 32 cases, there was no statistically significant result between total survival and cytoplasmic and nuclear survivin expressions when compared with the general group $(\mathrm{p}=0.769$ and $\mathrm{p}=0.347)$.
Table 3. The Statistical Assessment of the Cytoplasmic and Nuclear Survivin Results

\begin{tabular}{lcc}
\hline $\begin{array}{l}\text { The values of cytoplasmic and } \\
\text { nuclear surviving expression }\end{array}$ & $\begin{array}{c}\text { means/ } \\
\text { median }\end{array}$ & $\begin{array}{c}\text { SD/min } \\
\text {-max }\end{array}$ \\
\hline The spectrum of cytoplasmic survivin expression & 64.9 & 22.6 \\
The density of cytoplasmic survivin expression & 1.6 & 0.6 \\
The index ofcytoplasmic survivin expression & 4 & $(1-12)$ \\
The spectrum of nuclear survivin expression & 6.1 & 8.2 \\
The density of nuclear survivin expression & 2 & 0.5 \\
The index of nuclear survivin expression & 2 & $(0-6)$ \\
\hline
\end{tabular}

Table 4. The Relationships of Data Having Prognostic Importance with the Survival

\begin{tabular}{|c|c|c|}
\hline Prognostic varieties & OR & $P$ \\
\hline Age $(>55)$ & 1.96 & 0.243 \\
\hline Stage (33a) & 7.75 & 0.049 \\
\hline Grade & 1.24 & 0.737 \\
\hline 2-Mar & 1.05 & 0.945 \\
\hline $\begin{array}{l}\text { Preoperative hemoglobin } \\
\text { level }(<12)^{1}\end{array}$ & 1.37 & 0.472 \\
\hline $\begin{array}{l}\text { Preoperative platelet } \\
\text { level }(>307.000)^{1}\end{array}$ & 0.88 & 0.826 \\
\hline $\begin{array}{l}\text { Preoperative CA125 } \\
\text { level }(>35)\end{array}$ & 1.78 & 0.473 \\
\hline $\begin{array}{l}\text { The presence of lymph } \\
\text { node metastasis }\end{array}$ & 4.42 & 0.024 \\
\hline $\begin{array}{l}\text { The presence of paraaortic } \\
\text { lymph node metastasis }\end{array}$ & 4.03 & 0.015 \\
\hline $\begin{array}{l}\text { The presence of pelvic } \\
\text { lymph node metastasis }\end{array}$ & 4.27 & 0.016 \\
\hline Myometrial invasion & 4.4 & 0.155 \\
\hline Serosal invasion & 4.35 & 0.015 \\
\hline Cytology & 5.2 & 0.004 \\
\hline Lymph vascular space invasion & 6.4 & 0.016 \\
\hline Adnexal involvement & 2.29 & 0.136 \\
\hline $\begin{array}{l}\text { The presence of intra } \\
\text { abdominal metastasis }\end{array}$ & 8.57 & 0.001 \\
\hline $\begin{array}{l}\text { The presence of acid in } \\
\text { the first diagnosis }\end{array}$ & 4.79 & 0.042 \\
\hline Omentum involvement & 7.11 & 0.001 \\
\hline The presence of recurrence & 60.2 & 0.001 \\
\hline The index of cytoplasmic survivin & 0.97 & 0.798 \\
\hline The index of nuclear survivin & 0.93 & 0.776 \\
\hline
\end{tabular}

Mean recurrences time in the cases was computed as $87.32 \pm 5.79$. The relationships between non-diseased survival graphic of the cases was presented in Figure 2.

\section{Discussion}

Although endometrial cancer is the most common gynecologic cancer in the west world, it accounts for only $13 \%$ of the deaths depending on the gynecologic cancers. Binesh et al (2014) found in a study that survival of patients with EC is varied by stage and grade, and moderately varied by histology and age. Endometrial cancer develops the impairment of the sensitive balance between the cell proliferation and apoptosis due to the activation of oncogenes or the loss of tumor suppressor genes. But the genes causing this cancer and the relations among these genes have not completely yet been elucidated.

Survivin is a gene determined in recent years and is 
the member of apoptosis protein inhibitor (IAP) family inhibiting apoptosis in a different way since its members certain bc 1-2 family (Yuan et al., 2012). Apoptosis is the important event kept under control by various activator and inhibitor factors. But in cancer, there is an inhibition of apoptosis via anti apoptotic proteins. Unlike bcl-2 and other apoptosis inhibitor proteins (IAP), surviving is the protein which may not be identified in normal adult tissues, the differentiation of which was completed but is expressed in various types of cancers. It reveals its effect attaching to caspase with BIR (Baculovirus IAP Repeat) area existing in the other apoptosis inhibitor proteins (IAP). Survivin appears in G2-M phase of the cell cycle and associated with the microtubules of mitotic spindles within the special and satisfied reaction regulated by the microtubule dynamics. The extreme expression of survivin has on oncogenic effect. Because it may suppress the control point of $\mathrm{G} 2 / \mathrm{M}$ phase so that the cells progress toward the mitosis. In spite of its common expression in tumors, it has been stated that it would be valuable and new agent in the chemotherapy treatment based on the apoptosis of survivin ever since it is generally poorly expressed in the normal tissues.

There are few studies researching the prognostic importance of survivin in the cases with endometrial carcinoma. In the study including 31 cases with endometrial cancer by Takai et al (2002), the relation of survivin nuclear and cytoplasmic immunoreactivity with the clinical phase, histological grade, myometrial invasion and survival was investigated and found that it was correlated by bad prognosis. In the other study including 93 cases with endometrial carcinoma by Pallares et al (2005), there was no statistically relationship between survivin and histological type, grade, stage or survival. In the study including 50 cases with endometrial carcinoma by Erkanli et al (2007), the relationship of survivin with the prognostic factors such as stage, grade and myometrial invasion could not be identified in the similar way as in the study by Pallares et al. In addition, there was no significant relationship for the survival in both of these studies.

In our study, the expression of survivin, a member of an apoptosis inhibitor family whose effects are known on both the survival and prognosis and its localization in carcinogenesis in most cancer types, was investigated in the cases with endometrial carcinoma. In our study including 62 cases, the rates of survivin expression were obtained by the immune-histochemical investigation. Taking into account of the literature, cytoplasmic and nuclear expression range and density were assessed separately. Thanks to these results, the indexes of cytoplasmic and nuclear survivin were obtained by the index method strengthening and facilitating the assessment in immunehistochemical investigations. When investigated the relationship between total survival and clinical and surgical prognostic factors of the cases such as advance stage, the presence of lymph node metastasis (pelvic, paraaortic, pelvic and paraaortic), serosal involvement, cytology positivity, the presence of acid in the first diagnosis, lymph vascular space invasion, adnexal involvement, the presence of intra abdominal metastasis, omentum involvement and the presence of recurrence, the results obtained were statistically significant and effected the total survival negatively $(\mathrm{p}<0.05)$. The similar findings were obtained for non-diseased survival. When investigated the relationship between non-diseased survival and advanced stage lymph node metastasis (pelvic, paraaortic, pelvic and paraaortic), serosal involvement, cytology positivity, the presence of acid in the first diagnosis, lymph vascular space invasion, adnexal invaolvement, the presence of intra abdominal metastasis and omentum involvement, the results obtained were statistically significant and did not affect the non-diseased survival negatively $(\mathrm{p}<0.05)$. When investigated the relationship between non-diseased survival and the indexes of cytoplasmic nuclear survivin, the result was not statistically significant ( $p>0.05)$. In our study, the investigation of the advanced stage (stage $3 \mathrm{a}$ and above) cases as a subgroup increases the power of data obtained in identifying the effect on survival. As a result of the investigation on the advanced stage subgroup including 32 cases, the relationship between total survival and the indexes of cytoplasmic and nuclear survivin was not statistically significant in a similar way as in the results obtained in general group ( $\mathrm{p}=0.769$ and $\mathrm{p}=0.347$ ).

In addition, the relation between the indexes of cytoplasmic and nuclear surviving and all these prognostic factors was also investigated in our study. There was no statistically significant relationship between the indexes of cytoplasmic and nuclear detected for survivin and data such as age, body mass index, preoperative hemoglobin, platelet and CA125 levels, stage, grade, the presence of lymph node metastasis, the presence of pelvic or paraaortic lymph node metastasis, the number of metastatic lymph nodes (total, paraaortic and pelvic), myometrial invasion, serosal invasion, cytology result, lymph vascular space invasion, adnexal involvement, the presence of intraabdominal metastasis, the presence of acid in the first diagnosis, omentum involvement, adjuvant treatment management of the cases, the presence of recurrence and rate of mortality $(\mathrm{p}>0.05)$.

When assessed the results obtained in our study, there was no statistically relationship between survival or the prognostic factors such as grade and stage and the cytoplasmic and nuclear expression of surviving in endometrial carcinoma ( $\mathrm{p}>0.05)$. Our study results assist the findings obtained by Pallares et al (2005). Much attention has been given to the studies carried out to foresee the prognosis in many cancer types when the literature has been searched. It has been considered that the detection of the factors influencing directly the prognosis and functioning in carcinogenesis pathways as well as the general accepted prognostic factors will provide important facilities in identifying the treatment methods of the patients. The important problems such as the prediction of the course of the disease, especially the adjuvant treatment planning and identification of patient follow-up and frequencies will also be indicator. Especially over two decades, the treatments for target have been carried out. From the point of view of this, the detection of the factors in carcinogenesis pathways and their effects on prognosis and survival provide target for further studies. 


\section{References}

Baser E, Togrul C, Ozgu E, et al (2013). Sperm-associated antigen 9 is a promising marker for early diagnosis of endometrial cancer. Asian Pac J Cancer Prev, 14,7635-8.

Binesh F, Akhavan A, Behniafard N, et al (2014). Endometrial adenocarcinoma: clinicopathologic and survival characteristics in Yazd, Iran. Asian Pac J Cancer Prev, 15, 2797-801.

Cao XQ, Lu HS, Zhang L, et al (2014). MEKK3 and survivin expression in cervical cancer: association with clinicopathological factors and prognosis. Asian Pac J Cancer Prev, 15, 5271-6.

Damitri TD, Shamsuddin SH, Idris FM, et al (2014). Rapamycin and PF4 induce apoptosis by upregulating bax and downregulating survivin inMNU-induced breast cancer. Asian Pac J Cancer Prev. 15, 3939-44.

Erkanli S, Bolat F, Kayaselcuk F, et al (2007). COX-2 and survivin are overexpressed and positively correlated in endometrial carcinoma. Gynecol Oncol, 104, 320-325.

Felix AS, Weissfeld JL, Stone RA (2010). Factors associated with type I and type II endometrial cancer. Cancer Causes Control, 21, 1851.

Karami H, Baradaran B, Esfahani A, et al (2013). siRNAmediated silencing of survivin inhibits proliferation and enhances etoposide chemosensitivity in acute myeloid leukemia cells. Asian Pac J Cancer Prev, 14, 7719-24.

Lurain JR, Rice BL, Rademaker AW (1991). Prognostic factors associated with recurrence in clinical stage I adenocarcinoma of endometrium. Obstet. Gynecol, 78, 63-9.

Okada E, Murai Y, Matsui K, et al (2001). Survivin expression in tumour cell nuclei is predictive of a favorable prognosis ingastric cancer patients. Cancer Lett, 163, 109-16.

Pallares J, Martinez-Guitarte JL, Xavier D (2005). Survivin expression in endometrial carcinoma a tissue microarray study with correlation with PTEN and STAT-3. Int J Gynecol Pathol, 24, 247-53.

Rowlands IJ, Weinstein P, Nagle CM, et al (2011). Season of birth and risk of endometrial cancer. Asian Pac J Cancer Prev, 12, 1193-6.

Silverberg E, Boring C, Squires A (1990). Cancer statistics. CA Cancer J Clin, 90, 40.

Srivastava AK, Singh PK, Srivastava K, et al (2013). Diagnostic role of survivin in urinary bladder cancer. Asian Pac J Cancer Prev. 14, 81-5.

Takai N, Miyazaki T, Nishida M (2002). Survivin expression correlates with clinical stage, histological grade, invasive behavior and survival rate in endometrial carcinoma. Cancer Lett. 184, 105-16.

Yuan SF, Zhu LJ, Zheng WE, et al (2012). Expression of betatubulin III and survivin in advance stage breast cancer correlates with chemotherapeutic effects of docetaxel. Asian Pac J Cancer Prev, 13, 361-5. 